\title{
Two Lectures On Gauge Theory and Khovanov Homology
}

\author{
Edward Witten \\ School of Natural Sciences, Institute for Advanced Study \\ Einstein Drive, Princeton, NJ 08540 USA
}

\begin{abstract}
In the first of these two lectures, I use a comparison to symplectic Khovanov homology to motivate the idea that the Jones polynomial and Khovanov homology of knots can be defined by counting the solutions of certain elliptic partial differential equations in 4 or 5 dimensions. The second lecture is devoted to a description of the rather unusual boundary conditions by which these equations should be supplemented. An appendix describes some physical background. (Versions of these lectures have been presented at various institutions including the Simons Center at Stonybrook, the TSIMF conference center in Sanya, and also Columbia University and the University of Pennsylvania.)
\end{abstract}




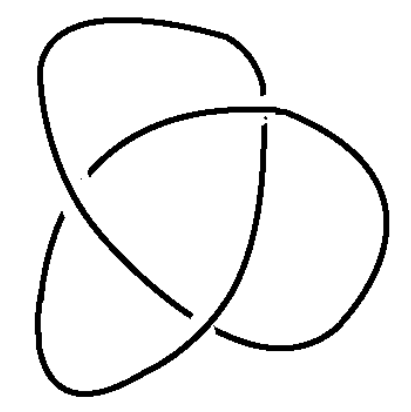

Figure 1: A knot embedded in $\mathbb{R}^{3}$.

\section{Lecture One}

The first physics-based proposal concerning Khovanov homology of knots was made by Gukov, Vafa, and Schwarz [1], who suggested that vector spaces associated to knots that had been introduced a few years earlier by Ooguri and Vafa [2] were related to what appears in Khovanov homology. A number of years later, I re-expressed this type of construction in terms of gauge theory and the counting of solutions of PDE's [3]. That is the story I will describe today. Several previous lectures are available $[4,5]$ (the second of these may be a better starting point) and I will take a different approach here.

In any event, the goal is to construct invariants of a knot embedded in $\mathbb{R}^{3}$ (fig. 1). In the simplest version, the invariants will be obtained by simply counting, with signs, the solutions of an equation. The solutions will have an integer-valued ${ }^{1}$ topological invariant $P$, and if $a_{n}$ is the "number" (counted algebraically) of solutions with $P=n$, then the Jones polynomial ${ }^{2}$ of the knot will be

$$
J(q)=\sum_{n} a_{n} q^{n}
$$

To get Khovanov homology, this situation is supposed to be "categorified," that is, we want for each $n$ to define a complex of vector spaces whose Euler characteristic is $a_{n}$. The only general situation that I know of in which one can naturally categorify the counting of solutions of an

\footnotetext{
${ }^{1}$ To be more precise, $P$ takes values in a $\mathbb{Z}$-torsor, rather than being canonically an integer. This is related to the framing anomaly of Chern-Simons theory. See Lecture 2.

${ }^{2}$ In approaches based on quantum field theory, the natural normalization of the Jones polynomial of a knot or link in $\mathbb{R}^{3}$ is such that the Jones polynomial of the empty link is 1. (The Jones polynomial is sometimes defined so that it equals 1 for an unknot rather than for the empty link.) We normalize the argument $q$ of the Jones polynomial to be the instanton counting parameter, in a sense that will be explained later. With this choice, the Jones polynomial of the unknot (with standard framing) is $q^{1 / 2}+q^{-1 / 2}$ and in general, for a knot with zero framing, the exponents in eqn. (1.1) are half-integers.
} 
equation is the case that the equation whose solutions we are counting describes the critical points of some Morse function $h$. We will be in this framework. Our equations will be partial differential equations or PDE's, so $h$ will be a Morse function on an infinite-dimensional space of functions, namely the functions that appear in the PDE. The categorification will involve a middle-dimensional cohomology theory of the function space, analogous to Floer theory. Let us put this aside for a moment and assume we are just trying to describe the uncategorified theory, that is the Jones polynomial.

The equations whose solutions I claim should be counted to define the Jones polynomial and ultimately Khovanov homology might look ad hoc if written down without an explanation of where they come from. I could have started today's lecture by explaining the physical setup, but this might be unhelpful for some. I decided instead to try a different approach of motivating the equations by comparing to an established mathematical approach to Khovanov homology, namely symplectic Khovanov homology [6-8].

Going all the way back to the original work of Vaughn Jones [9], most approaches to the Jones polynomial define an invariant in terms of some sort of presentation of a knot, for example a projection to a plane - such as the projection used in drawing fig. 1. One defines something that is manifestly well-defined and explicitly computable once such a presentation is given. What one defines is not obviously independent of the knot presentation, but turns out to be. That step is where the magic is. And there is always some magic.

An approach based on counting solutions of PDE's has the opposite advantages and drawbacks. Topological invariance is potentially manifest (given certain generalities about elliptic PDE's and assuming compactness is under control), but it may not be clear how to calculate. The ideal is to have manifest three- or (in the categorified case) four-dimensional symmetry together with a method of calculation. How might this be achieved?

I will suggest how to guess the right equations starting from a knowledge of symplectic Khovanov homology. But in order to do this, we need to know something about a possible strategy to actually count the solutions of an equation. So I will begin by explaining what we would do if we knew which equations we want to analyze, and this will help us in guessing the equations.

There is a standard strategy, applicable to the present problem, for trying to count solutions of a PDE under suitable conditions. The original version was the Atiyah-Floer conjecture concerning Floer homology of a three-manifold [10]. Adapting their approach to the present problem, the idea is to stretch a knot in one direction, say the $u$ direction, as in fig. 2. Then one wants it to be the case that except near the ends, the solutions are independent of $u$. This is not automatically the case and in [11], where this strategy was followed for the present problem, it was necessary to make a perturbation to a more generic system of equations to get to a situation in which this would be true.

Given this, we define a moduli space $\mathcal{M}$ of $u$-independent solutions. We can think of these as the solutions in the presence of infinite parallel strands that run in the $u$ direction, as in fig. 3 . 


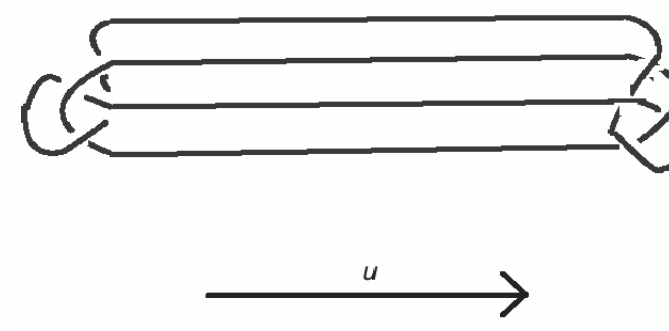

Figure 2: A knot embedded in $\mathbb{R}^{3}$ and stretched in one direction.
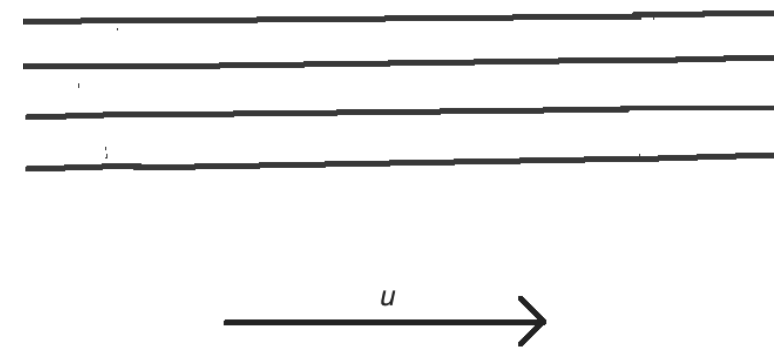

Figure 3: Infinite parallel strands parametrized by $u$, with $-\infty \leq u \leq \infty$.
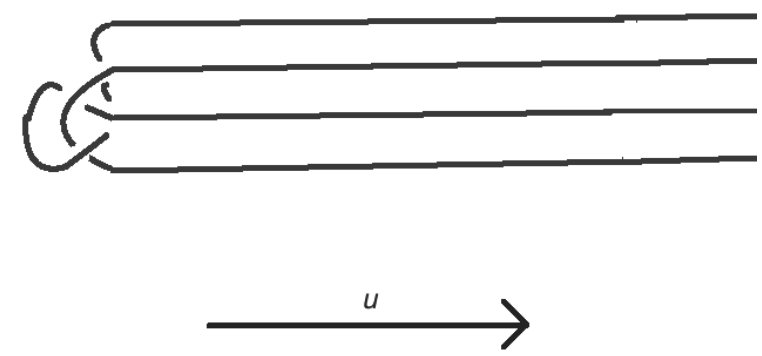

Figure 4: Semi-infinite strands that extend to $u=+\infty$. 
Now as in fig. 4 consider solutions in the presence of semi-infinite strands that extend to $u=+\infty$ or to $u=-\infty$ but not both. Let $\mathcal{L}_{\ell}$ and $\mathcal{L}_{r}$ be the moduli spaces of such solutions. Thus a point in $\mathcal{L}_{\ell}$ represents a solution in a semi-infinite situation in which the strands terminate on the left (as drawn in fig. 4). Likewise $\mathcal{L}_{r}$ parametrizes solutions in the presence of semi-infinite strands that terminate on the right. We assume that a solution in such a semi-infinite situation is independent of $u$ for $u \rightarrow+\infty$ or $u \rightarrow-\infty$, respectively. If this is so, then $\mathcal{L}_{\ell}$ and $\mathcal{L}_{r}$ come with natural maps to $\mathcal{M}$. For simplicity in our terminology, we will assume that these maps are embeddings; this amounts to assuming that each solution in the interior in fig. 2 can be extended over the left or over the right in at most one way. This assumption is not necessary but makes the explanation simpler.

The solutions for a global knot like the one in fig. 2 can be understood as solutions in the middle that extend over both ends. So the global solutions are intersection points of $\mathcal{L}_{\ell}$ and $\mathcal{L}_{r}$. The integer $a_{n}$ that appears as a coefficient in the Jones polynomial is supposed to be the algebraic intersection number of $\mathcal{L}_{\ell}$ and $\mathcal{L}_{r}$ :

$$
a_{n}=\mathcal{L}_{\ell} \cap \mathcal{L}_{r}
$$

(To be more exact, $a_{n}$ is this intersection number computed by counting only intersections with $P=n$.)

In this language of intersections, categorification can happen if $\mathcal{M}$ is in a natural way a symplectic manifold and $\mathcal{L}_{\ell}$ and $\mathcal{L}_{r}$ are Lagrangian submanifolds. Then Floer cohomology - i.e. the $A$-model or the Fukaya category - of $\mathcal{M}$ gives a framework for categorification. From the point of view of today's lecture, the reason that all this will happen is that, even before we stretched the knot to reduce to intersections in $\mathcal{M}$, the equations whose solutions we were counting are equations for critical points of some Morse function(al) $h$.

In "symplectic Khovanov homology," a version of such a story is developed for Khovanov homology (at least in a singly-graded version) with a very specific $\mathcal{M}$. A description of this $\mathcal{M}$ that was proposed in [12] (and exploited in a mirror version in [13]) and which provided an important clue in my work is as follows. $\mathcal{M}$ can be understood as a space of Hecke modifications. Let me explain this concept. Let $C$ be a Riemann surface and $E \rightarrow C$ a holomorphic $G_{\mathbb{C}}$ bundle over $C$, where $G_{\mathbb{C}}$ is some complex Lie group. A Hecke modification of $E$ at a point $p \in C$ is a holomorphic $G_{\mathbb{C}}$ bundle $E^{\prime} \rightarrow C$ with an isomorphism to $E$ away from $p$ :

$$
\varphi:\left.\left.E^{\prime}\right|_{C \backslash p} \cong E\right|_{C \backslash p} .
$$

For example, if $G_{\mathbb{C}}=\mathbb{C}^{*}$, the we can think of $E$ as a holomorphic line bundle $\mathcal{L} \rightarrow C$. A holomorphic bundle $\mathcal{L}^{\prime}$ that is isomorphic to $\mathcal{L}$ away from $p$ is

$$
\mathcal{L}^{\prime}=\mathcal{L}(n p)=\mathcal{L} \otimes \mathcal{O}(p)^{n}
$$

for some integer $n$. Here $n$ can be thought of as a weight of the Langlands-GNO dual group of $\mathbb{C}^{*}$, which is another copy of $\mathbb{C}^{*}$.

The reason that I write $G_{\mathbb{C}}$, making explicit that this is the complex form of the group, is that when we do gauge theory, the gauge group will be the compact real form and I will call this 


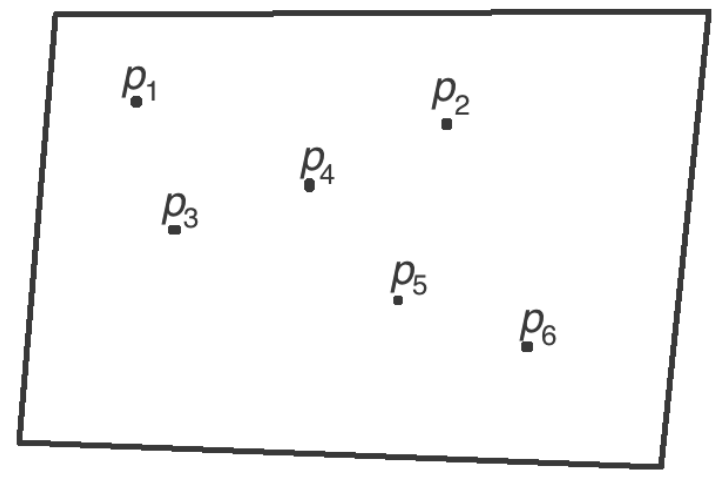

Figure 5: A configuration of points $p_{i} \in \mathbb{R}^{2}$, at which we are going to make Hecke modifications.

simply $G$. In general, for any $G$, there is a corresponding Langlands-GNO dual group $G^{\vee}$, with

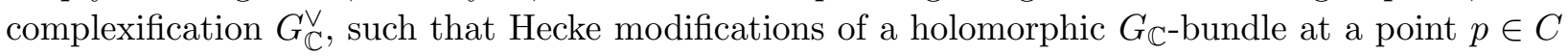
occur in families classified by dominant weights (or equivalently finite-dimensional representations) of $G_{\mathbb{C}}^{\vee}$ (or equivalently $G^{\vee}$ ).

For example, if $G_{\mathbb{C}}=G L(2, \mathbb{C})$, we can think of a $G_{\mathbb{C}^{-}}$-bundle $E \rightarrow C$ as a rank 2 complex vector bundle $E \rightarrow C$. The Langlanda-GNO dual group $G_{\mathbb{C}}^{\vee}$ is again $G L(2, \mathbb{C})$, and a Hecke modification dual to the 2-dimensional representation of $G_{\mathbb{C}}^{\vee}$ is as follows. For some local decomposition $E \cong$ $\mathcal{O} \oplus \mathcal{O}$ in a neighborhood of $p \in C$, one has $E^{\prime} \cong \mathcal{O}(p) \oplus \mathcal{O}$. The difference from the abelian case is that there is not just one Hecke modification of this type at $p$ but a whole family of them, arising from the choice of a subbundle $\mathcal{O}$ of $E$ that is going to be replaced by $\mathcal{O}(p)$.

Because of this dependence, the Hecke modifications of this type at $p$ form a family, parametrized by $\mathbb{C P}^{1}$. Suppose we are given $2 n$ points on $\mathbb{C} \cong \mathbb{R}^{2}$ at which we are going to make Hecke modifications of this type of a trivial bundle rank 2 complex vector bundle $E \rightarrow \mathbb{C}$ (fig. 5). The space of all such Hecke modifications would be a copy of $\left(\mathbb{C P}^{1}\right)^{2 n}$, with one copy of $\mathbb{C P}^{1}$ at each point. However, there is a natural subvariety $\mathcal{M} \subset\left(\mathbb{C P}^{1}\right)^{2 n}$ defined as follows. One adds a point $\infty$ at infinity to compactify $\mathbb{C}$ to $\mathbb{C P}^{1}$, so we are now making Hecke modifications of a trivial bundle $E=\mathcal{O} \oplus \mathcal{O} \rightarrow \mathbb{C P}^{1}$. A point in $\left(\mathbb{C P}^{1}\right)^{2 n}$ determines a way to perform Hecke modifications at the points $p_{1}, p_{2}, \ldots, p_{2 n}$ to make a new bundle $E^{\prime}$. The space $\mathcal{M}$ is defined by requiring that $E^{\prime} \otimes \mathcal{O}(-n \infty)$ is trivial. (If we were working in $P G L(2, \mathbb{C})$ rather than $G L(2, \mathbb{C}$ ), we would just say that $E^{\prime}$ should be trivial.)

Symplectic Khovanov homology is constructed by considering intersections of Lagrangian submanifolds of the space $\mathcal{M}$ of multiple Hecke modifications from a trivial bundle to itself. We want to reinterpret this in terms of gauge theory PDE's.

In my work with Kapustin on gauge theory and geometric Langlands [15], an important fact was that $\mathcal{M}$ can be realized as a moduli space of solutions of a certain system of PDE's. However, 
although $\mathcal{M}$ is defined in terms of bundles on a 2 -manifold $\mathbb{R}^{2} \cong \mathbb{C}$, the PDE's are in 3 dimensions - on $\mathbb{R}^{3}$. As a result of this, everything in the rest of the lecture will be in a dimension one more than one might expect. To describe the Jones polynomial - an invariant of knots in 3-space - we will count solutions of certain PDE's in 4 dimensions, and the categorified version - Khovanov homology - will involve PDE's in 5 dimensions.

The 3-dimensional PDE's that we need are known as the Bogomolny equations. They are equations, on an oriented three-dimensional Riemannian manifold $W_{3}$, for a pair $A$, $\phi$, where $A$ is a connection on a $G$-bundle $E \rightarrow W_{3}$, and $\phi$ is a section of $\operatorname{ad}(E) \rightarrow W_{3}$ (i.e. an adjoint-valued 0 -form). If $F=\mathrm{d} A+A \wedge A$ is the curvature of $A$, then the Bogomolny equations are

$$
F=\star \mathrm{d}_{A} \phi .
$$

(Here $\star$ is the Hodge star and $\mathrm{d}_{A}$ is the gauge-covariant extension of the exterior derivative.)

The Bogomolny equations have many remarkable properties and we will focus on just one aspect. We consider the Bogomolny equations on $W_{3}=\mathbb{R} \times C$ with $C$ a Riemann surface. Any connection $A$ on a $G$-bundle $E \rightarrow C$ determines a holomorphic structure on $E$ (or more exactly on its complexification): one simply writes $\mathrm{d}_{A}=\bar{\partial}_{A}+\partial_{A}$ and uses $\bar{\partial}_{A}$ to define the complex structure. (In complex dimension 1 , there is no integrability condition that must be obeyed by a $\bar{\partial}$ operator.) So for any $y \in \mathbb{R}$, by restricting $E \rightarrow \mathbb{R} \times C$ to $E \rightarrow\{y\} \times C$, we get a holomorphic bundle $E_{y} \rightarrow C$. However, if the Bogomolny equations are satisfied, $E_{y}$ is canonically independent of $y$. Indeed, a consequence of the Bogomolny equations is that $\bar{\partial}_{A}$ is independent of $y$ up to conjugation. If we parametrize $\mathbb{R}$ by $y$, then the Bogomolny equations imply that

$$
\left[\frac{D}{D y}-\mathrm{i} \phi, \bar{\partial}_{A}\right]=0
$$

Thus $\bar{\partial}_{A}$ is independent of $y$, up to a natural conjugation.

The Bogomolny equations admit solutions with singularities at isolated points. To understand the basic picture, we take the three-manifold to be simply $\mathbb{R}^{3}$, and the gauge group to be $U(1)$. One fixes an integer $n$ and one observes that the Bogomolny equation has an exact solution for any $x_{0} \in \mathbb{R}^{3}$ :

$$
\phi=\frac{n}{2\left|\vec{x}-\vec{x}_{0}\right|}, \quad F=\star \mathrm{d} \phi .
$$

I have only defined $F$ and not the connection $A$ whose curvature is $F$ or the line bundle $\mathcal{L}$ on which $A$ is connection. Such an $\mathcal{L}$ and $A$ exist (and are essentially unique) if and only if $n \in \mathbb{Z}$.

For $G=U(1)$, since the Bogomolny equations are linear, they have a unique solution with singularities of this type labeled by specified integers $n_{1}, n_{2}, \ldots$ at specified points $p_{i} \in \mathbb{R}^{3}$ (fig. 6). We simply take $\phi=\sum_{i} \frac{n_{i}}{2\left|\vec{x}-\vec{x}_{i}\right|}, F=\star \mathrm{d} \phi$. We assume that $\sum_{i} n_{i}=0$, which ensures that $\phi$ and the connection $A$ vanish at infinity faster than $1 /|\vec{x}|$.

Now pick a decomposition $\mathbb{R}^{3}=\mathbb{R} \times \mathbb{R}^{2}$, where we identify $\mathbb{R}^{2}$ as $\mathbb{C}$. Suppose that the singularities are at $y_{i} \times p_{i}$, with $y_{i} \in \mathbb{R}, p_{i} \in \mathbb{C}$. For each $y \notin\left\{y_{1}, \ldots, y_{n}\right\}$, the indicated solution of the Bogomolny 


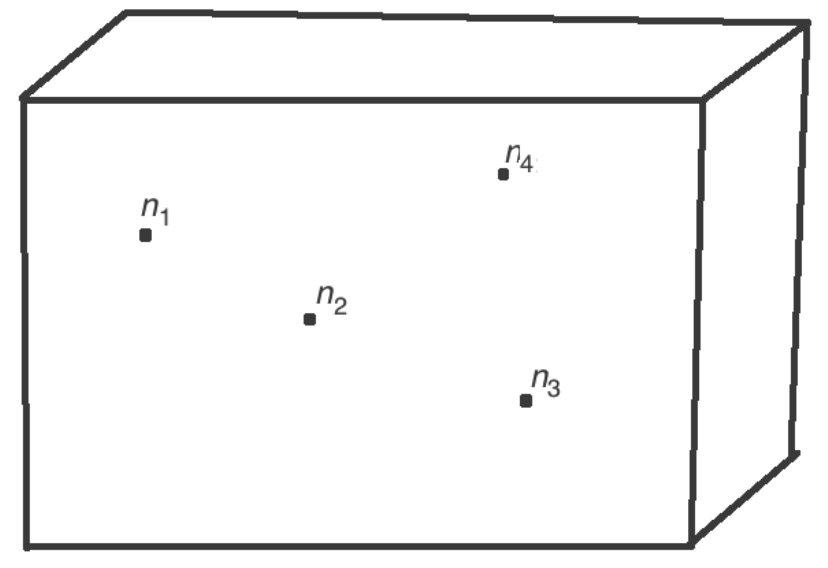

Figure 6: Points $y_{i} \times p_{i} \in \mathbb{R}^{3}$ labeled by weights $n_{i}$ of the group $U(1)$.

equations determines a holomorphic line bundle $\mathcal{L}_{y} \rightarrow \mathbb{C}$, and upon adding a point at infinity, this naturally extends to $\mathcal{L}_{y} \rightarrow \mathbb{C P}^{1}$. (Here we use the fact that $A$ vanishes at infinity faster than $1 /|\vec{x}|$.) $\mathcal{L}_{y}$ is independent of $y$ up to isomorphism as long as $y$ is not equal to one of the $y_{i}$, but even when $y$ crosses one of the $y_{i}, \mathcal{L}_{y}$ is constant when restricted to $\mathbb{C P}^{1} \backslash p_{i}$. In crossing $y=y_{i}, \mathcal{L}_{y}$ undergoes a Hecke modification

$$
\mathcal{L}_{y} \rightarrow \mathcal{L}_{y} \otimes \mathcal{O}\left(p_{i}\right)^{n_{i}}
$$

$\mathcal{L}_{y}$ is trivial for $y \rightarrow-\infty$ and for $y \rightarrow+\infty$ (again because the solution vanishes at infinity faster than $1 /|\vec{x}|)$. The solution thus describes a sequence of Hecke modifications mapping the trivial bundle to itself.

We can do something similar for any simple Lie group $G$. (The underlying idea was introduced by 't Hooft in the late 1970's [14] and is important in physical applications of quantum gauge theory.) Let $T$ be the maximal torus of $G$ and let $\mathfrak{t}$ be its Lie algebra. Pick a homomorphism $\rho: \mathfrak{u}(1) \rightarrow \mathfrak{t}$. Up to a Weyl transformation, such a $\rho$ is equivalent to a dominant weight of the dual group $G^{\vee}$, so it corresponds to a representation $R^{\vee}$ of $G^{\vee}$. We turn the singular solution (1.7) of the $U(1)$ Bogomolny equations that we already used (more exactly, the special case of this solution with $n=1$ ) into a singular solution for $G$ simply by

$$
(A, \phi) \rightarrow(\rho(A), \rho(\phi)) .
$$

Then we look for solutions of the Bogomolny equations for $G$ with singularities of this type at specified points $y_{i} \times p_{i} \in \mathbb{R}^{3}$.

The picture is the same as before except that now (fig. 7) the points $y_{i} \times p_{i}$ are labeled by homomorphisms $\rho_{i}: \mathfrak{u}(1) \rightarrow \mathfrak{t}$, or in other words by representations $R_{i}^{\vee}$ of the dual group $G^{\vee}$, rather than by integers $n_{i}$. Also, now we must specify that the solution should go to 0 at infinity faster than $1 / r$ (for $U(1)$, this was automatic once we set $\left.\sum_{i} n_{i}=0\right)$. Given this, such a solution 


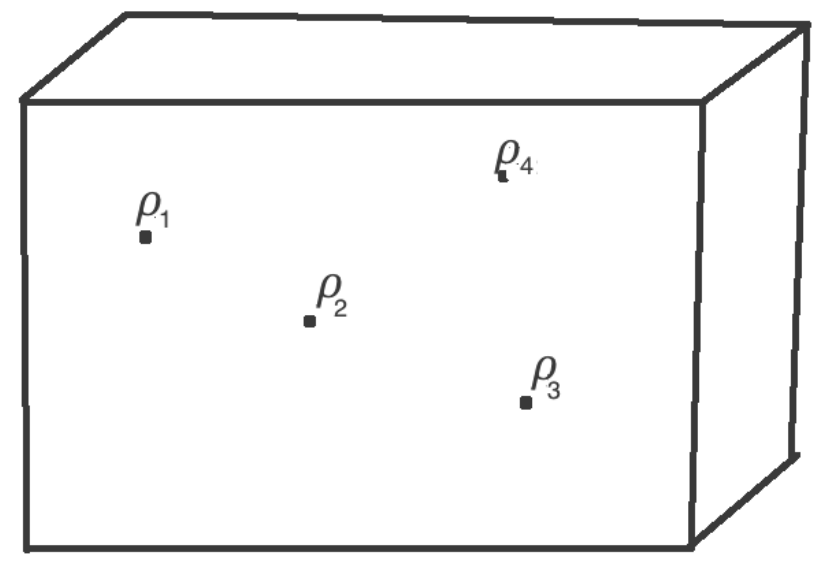

Figure 7: Points in $\mathbb{R}^{3}$ labeled by homomorphisms $\rho_{i}: \mathfrak{u}(1) \rightarrow \mathfrak{t}$, or equivalently by representations of the dual group.

describes a sequence of Hecke modifications at $p_{i}$ of type $\rho_{i}$, mapping a trivial $G$-bundle $E \rightarrow \mathbb{C P}^{1}$ to itself.

The moduli space $\mathcal{M}$ of solutions of the Bogomolny equations on $\mathbb{R}^{3}$ with the indicated singularities and vanishing at infinity faster than $1 / r$ is actually a hyper-Kahler manifold, essentially first studied by P. Kronheimer in the 1980's. If we pick a decomposition $\mathbb{R}^{3}=\mathbb{R} \times \mathbb{R}^{2}$, this picks one of the complex structures on the hyper-Kahler manifold and in that complex structure, $\mathcal{M}$ is the moduli space $\mathcal{M}_{p_{1}, \rho_{1} ; p_{2}, \rho_{2} ; \ldots}$ of all Hecke modifications of the indicated types at the indicated points, mapping a trivial bundle over $\mathbb{C P}^{1}$ to itself.

This construction can be used to account for a number of properties of spaces of Hecke modifications, but for today we want to focus on the application to knot theory. The reduction to $\mathcal{M}$ is supposed to result from stretching a knot in one direction, so we want $\mathcal{M}$ to be the space of $u$-independent solutions of some equations, as suggested in fig. 3. We already described $\mathcal{M}$ via solutions of some PDE's on $\mathbb{R}^{3}$, so now we have to think of $\mathcal{M}$ as a space of $u$-independent solutions on $\mathbb{R}^{4}=\mathbb{R}^{3} \times \mathbb{R}$, where the second factor is parametrized by $u$.

There actually are natural PDE's in four dimensions that work. They play a role in the gauge theory approach to geometric Langlands [15], and are sometimes called the KW equations. They are equations for a pair $A, \phi$ where $A$ is a connection on $E \rightarrow Y_{4}, Y_{4}$ a four-manifold, and $\phi$ is a 1-form on $Y_{4}$ valued in $\operatorname{ad}(E)$ :

$$
F-\phi \wedge \phi=\star \mathrm{d}_{A} \phi, \quad \mathrm{d}_{A} \star \phi=0 .
$$

In a special case $Y_{4}=W_{3} \times \mathbb{R}$, with $A$ a pullback from $W_{3}$ and $\phi=\phi \mathrm{d} u$ (where $\phi$ is a section of $\operatorname{ad}(E)$ and $u$ parametrizes the second factor in $Y_{4}$ ) these equations reduce to the Bogomolny 


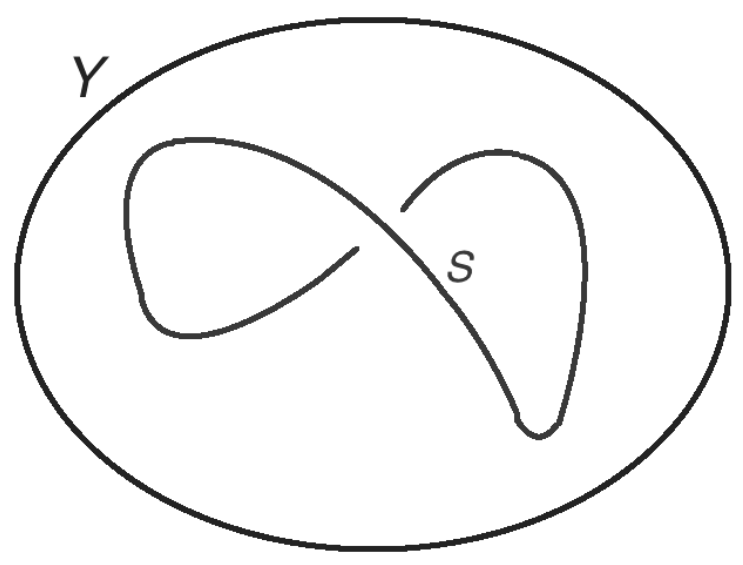

Figure 8: A four-manifold $Y$ with an embedded 1-manifold $S$ along which one specifies a desired singularity.

equations on $W_{3}$ :

$$
F=\star \mathrm{d}_{A} \phi .
$$

Therefore, the singular solution (1.9) of the Bogomolny equations that we have already studied can be lifted to a singular solution of the KW equations, but now the singularity is along a line rather than a point. Of course, the singularity is still in codimension three. We view this solution as a model that tells us what sort of codimension three singularity to look for in a more general situation. If $Y_{4}$ is a 4-manifold and $S \subset Y_{4}$ is an embedded 1-manifold, labeled by a homomorphism $\rho: \mathfrak{u}(1) \rightarrow \mathfrak{t}$ (or by a representation of $G^{\vee}$ ), then one can look for solutions of the KW equations with a singularity along $S$ associated to the given choice of $\rho$ (fig. 8).

If we specialize to the case that $Y_{4}=\mathbb{R}^{3} \times \mathbb{R}$, with $S=\cup_{i} S_{i}$, and $S_{i}=p_{i} \times \mathbb{R} \subset \mathbb{R}^{3} \times \mathbb{R}\left(p_{i}\right.$ are points in $\mathbb{R}^{3}$ and $\mathbb{R}$ is parametrized by $u$ ) then the $u$-independent solutions of the KW equations are just the solutions of the Bogomolny equations on $\mathbb{R}^{3}$, with the chosen singularities. So these solutions are parametrized by $\mathcal{M}$; and indeed one can show that these are all solutions of the $\mathrm{KW}$ equations in this situation with reasonable behavior at infinity.

So we have an elliptic PDE in four dimensions and we can specify in an interesting way what sort of singularity it should have on an embedded circle $S \subset Y_{4}$. But this sounds like a ridiculous framework for knot theory, because there is no knottedness of a 1-manifold in a 4-manifold!

To resolve this point, we have to explain what is involved in categorification. Let us practice with an ordinary equation rather than a partial differential equation. Suppose that we are on a finite-dimensional compact oriented manifold $N$ with a real vector bundle $V \rightarrow N$ with $\operatorname{rank}(V)=\operatorname{dimension}(N)$. Suppose also we are given a section $s$ of $V$. We can define an integer by counting, with multiplicities (and in particular with signs) the zeroes of $s$. This integer is the Euler class $\int_{M} \chi(V)$. 
Figure 9: A flow from one critical point to another.

In general as far as I know, there is no way to categorify the Euler class of a vector bundle. However, suppose that $V=T^{*} N$ and that $s=\mathrm{d} h$ where $h$ is a Morse function. Then the zeroes of $s$, which are critical points of $h$, have a natural "categorification" described in Morse homology. One defines a complex $\mathcal{V}$ with a basis vector $\psi_{p}$ for each critical point $p$ of $h$. The complex is $\mathbb{Z}$-graded by assigning to $\psi_{p}$ the "index" of the critical point $p$, and it has a natural differential that is defined by counting gradient flow lines between different critical points.

Concretely the differential is defined by

$$
\mathrm{d} \psi_{p}=\sum_{q} n_{p q} \psi_{q}
$$

where the sum runs over all critical points $q$ whose Morse index exceeds by 1 that of $\mathrm{p}$, and the integer $n_{p q}$ is defined by counting flows from $p$ to $q$ (fig. 9). A "flow" is a solution of the gradient flow equation

$$
\frac{\mathrm{d} \vec{x}}{\mathrm{~d} t}=-\vec{\nabla} h .
$$

(To define this equation, one has to pick a Riemannian metric on the manifold $N$. The complex that one gets is independent of the metric up to quasi-isomorphism. One considers flows that start at $p$ at $t=-\infty$ and end at $q$ at $t=+\infty$. Such flows come in one-parameter families related by time translations and $n_{p q}$ is the number of such families, counted algebraically.)

This tells us what we need in order to be able to categorify a problem of counting solutions of the KW equations. We have to be able to write those equations as equations for a critical point of a functional $\Gamma(A, \phi)$ :

$$
\frac{\delta \Gamma}{\delta A}=\frac{\delta \Gamma}{\delta \phi}=0 .
$$

And the associated gradient flow equation, which will be a PDE in 5 dimensions on $X_{5}=\mathbb{R} \times Y_{4}$

$$
\frac{\mathrm{d} A}{\mathrm{~d} t}=-\frac{\delta \Gamma}{\delta A}, \quad \frac{\mathrm{d} \phi}{\mathrm{d} t}=-\frac{\delta \Gamma}{\delta \phi}
$$


has to be elliptic, so that it will makes sense to try to count its solutions.

Generically, it is not true that the KW equations on a manifold $Y_{4}$ are equations for a critical point of some functional. However, this is true if $Y_{4}=W_{3} \times \mathbb{R}$ for some $W_{3}$. If singularities are present on an embedded 1-manifold $S \subset Y_{4}$ then there is a further condition: The KW equations in this situation are equations for critical points of a functional if and only if $S$ is contained in a 3 -manifold $W_{3} \times p$, with $p$ a point in $\mathbb{R}$. (For an explanation of "why" this is true, see the appendix.) So to make categorification possible, we have to be in the situation that leads to knot theory: $S$ is an embedded 1-manifold in a 3-manifold $W_{3}$. Once this restriction is made, the five-dimensional flow equations exist and are indeed elliptic. (They were introduced independently in [3] and [19] and are sometimes called the HW equations.)

Naively, this leads to "categorified" knot invariants for any three-manifold $W_{3}$, but to justify this claim one needs some compactness properties for solutions of the equations under consideration. I suspect that a proper proof of these compactness properties may require that the Ricci tensor of $W_{3}$ is nonnegative, a very restrictive condition.

What I have described so far is supposed to correspond (for $W_{3}=\mathbb{R}^{3}, G=S O(3)$ and $\rho$ corresponding to the 2-dimensional representation of $\left.G^{\vee}=S U(2)\right)$ to "singly-graded Khovanov homology." It is singly-graded because the only grading I have mentioned is the grading that is associated to the Morse index, or in other words to categorification. In the mathematical theory, one says that singly-graded Khovanov homology becomes trivial (it does not distinguish knots) if one "decategorifies" it and forgets the grading. In the approach I have described, this is true because in the uncategorified version, the embedded 1-manifold $S$ is just a 1-manifold in a 4-manifold $Y_{4}$ (it has no reason to be embedded in the 3 -manifold $W_{3} \times p$ ) so there is no knottedness.

The physical picture makes clear where the additional " $q$ "-grading of Khovanov homology would come from. It is supposed to come from the second Chern-class, integrated over the 4-manifold $Y_{4}$. The second Chern class is the invariant that I called $P$ at the beginning of the lecture. But for topological reasons, the second Chern class cannot be defined in the presence of a codimension three singularity of the type I have described on an embedded one-manifold $S \subset Y_{4}$. (Because of the singularity, $Y_{4}$ behaves as a noncompact four-manifold on which there is no topological invariant corresponding to the second Chern class of a $G$ bundle.) And therefore the construction as I have presented it so far has no $q$-grading.

The physical picture tells us what we have to do to get the $q$-grading: $Y_{4}$ should be a manifold with boundary, with the knot placed in its boundary (fig. 10). The appropriate boundary condition will be the subject of Lecture Two and is such that the second Chern class can be defined.

In [11], Gaiotto and I analyzed this problem (in the uncategorified situation, meaning that we counted solutions in 4 dimensions, not 5, and for the simplest case of $G=S O(3)$ ) with the aim of showing directly, without referring to the physical picture, that the Jones polynomial is

$$
J(q)=\sum_{n} a_{n} q^{n}
$$




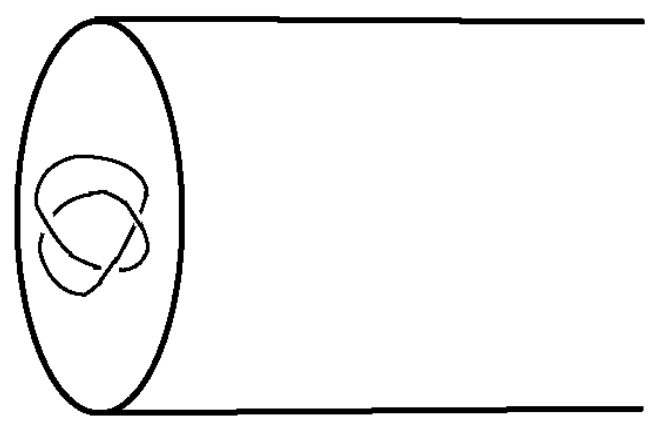

Figure 10: A four-manifold $Y_{4}$ with boundary, with a knot placed in its boundary.

where $a_{n}$ is the number of solutions with second Chern class $n$. The starting point was to stretch the knot in one direction, reducing to equations in one dimension less, as in fig. 2. It turns out that the solutions in one dimension less that satisfy the boundary condition are related to a lot of interesting mathematical physics involving integrable systems, conformal field theory, geometric Langlands, and more. What emerges is the "vertex model" construction of the Jones polynomial; the way it emerges is somewhat along the lines of work by Bigelow [16] and Lawrence [17]. What our work added was a derivation of the vertex model from a starting point with manifest 3-dimensional symmetry. The analog of this for the categorified theory is expected to involve, in one version, a Fukaya-Seidel category with a certain superpotential. The relevant model - as well as a plausible variant that does not work - has been explored and to a considerable extent understood in [18].

\section{Lecture Two}

In Lecture One, I explained that to define a $q$-grading in Khovanov homology, we have to be able to make sense of the second Chern class of a solution of the KW equations on a four-manifold $Y_{4}$, in the presence of a knot. As already explained, if the knot is represented by a codimension 1 embedded submanifold $S \subset Y_{4}$, this will not work, because the singularity that we want to postulate along $S$ does not allow the definition of a second Chern class as a topological invariant. Instead, we embed the knot in the boundary of $Y_{4}$, as in fig. 10. The boundary condition that we use is subtle to describe, but has the property that the bundle is fixed on the boundary, so the second Chern class can be defined.

We could actually get the $q$-grading for any $Y_{4}$ with boundary, but to also allow categorification, we want more specifically $Y_{4}=W \times \mathbb{R}_{+}$, where $\mathbb{R}_{+}$is a half-line, parametrized by $y$. For the Jones polynomial and Khovanov homology, we further take $W=\mathbb{R}^{3}$. (More general choices of $W$ are certainly also interesting, but not much is known about what to expect. See [20].) So as sketched 
in fig. $10, Y_{4}$ is $\mathbb{R}^{3} \times \mathbb{R}_{+}$with the knot embedded in the boundary.

For $y \rightarrow \infty$, we ask for $A, \phi \rightarrow 0$. For $y \rightarrow 0$, there is a subtle boundary condition which is one of the main points of the theory. Describing it is actually my main goal for today. This boundary condition depends on the knot $K$, and on the labeling of $K$ (or of each component of a link $L$ ) by a representation $R^{\vee}$ of the Langlands or GNO dual group $G^{\vee}$. That is the only place that $K$ enters the setup.

The desired boundary condition is an elliptic boundary condition though possibly an unexpected one. Here, "elliptic" means that although the definition may look unexpected, the resulting properties are similar to what one would get with more familiar elliptic boundary conditions such as Dirichlet or Neumann. For example, on a compact four-manifold, the linearization of the KW equation becomes a Fredholm operator and has discrete spectrum.

With this boundary condition, which I will describe in some detail, the restriction to the boundary $W \times\{y=0\}$ of the bundle $E$ and connection $A$ are specified. As a result, one can define a second Chern class

$$
n=\frac{1}{8 \pi^{2}} \int_{W \times \mathbb{R}_{+}} \operatorname{Tr} F \wedge F .
$$

However, because the bundle is fixed on the boundary but is not trivialized, this invariant really takes values in a $\mathbb{Z}$-torsor associated to framings of $W$ and $K$. "Torsor" means that it is not true that $n$ is an integer in a canonical way; rather the value of $n \bmod \mathbb{Z}$ depends only on the boundary conditions and not on the specific gauge field that satisfies them. One can "trivialize the torsor" and make $n$ an integer by picking framings of $W$ and $K$.

To define a knot polynomial, one counts (with signs, in a standard way) the number $a_{n}$ of solutions of the KW equations with second Chern class $n$, and then one defines

$$
J\left(q ; K, R^{\vee}\right)=\sum_{n \in \mathbb{Z}} a_{n} q^{n} .
$$

Compactness (not yet proved with the appropriate boundary conditions) of the solutions of the KW equations will mean that there are only finitely many terms in the sum so that this is a Laurent polynomial.

As I explained in Lecture One, with this definition of the Jones polynomial, the "categorification" that leads to Khovanov homology is straightforward in principle. It arises because the $\mathrm{KW}$ equations can be "lifted," in a certain sense, to certain elliptic differential equations in five dimensions, and these equations can be interpreted as gradient flow equations. But rather than say more about that today, what I want to do is to describe the boundary condition that is needed for the four (or five) dimensional equations. This boundary condition is of a possibly somewhat unfamiliar type, and understanding it is essential for making progress with this subject.

The boundary conditions have been studied in [21], and were shown to be elliptic in the absence of a knot. A paper is in progress on the case with a knot [22], and I will tell a little about that 
case later. I will carry out this discussion in the language of the four-dimensional equations, since going to five dimensions does not change much, as was shown in [21].

The boundary condition of interest is not a simple Dirichlet or Neumann boundary condition - it is not defined by saying what fields or derivatives of fields vanish along the boundary. Rather, the boundary condition is defined by specifying a model solution of the KW equations that has a singularity along the boundary, and saying that one only wants to consider solutions of the KW equation that are asymptotic to this singular solution along the boundary.

The model solution is a solution on $\mathbb{R}^{3} \times \mathbb{R}_{+}$, where I will parametrize $\mathbb{R}^{3}$ by $x_{1}, x_{2}, x_{3}$ and $\mathbb{R}_{+}$ by $y$. There is a simple exact solution with $A=0$ and

$$
\phi=\sum_{i=1}^{3} \frac{\mathfrak{t}_{i} \cdot \mathrm{d} x_{i}}{y},
$$

where $\mathfrak{t}_{i}$ are elements of the Lie algebra $\mathfrak{g}$ of $G$ that obey the $\mathfrak{s u}(2)$ commutation relations

$$
\left[\mathfrak{t}_{1}, \mathfrak{t}_{2}\right]=\mathfrak{t}_{3} \text {, and cyclic permutations. }
$$

Thus the $\mathfrak{t}_{i}$ are images of a standard basis of $\mathfrak{s u}(2)$ under some homomorphism $\rho: \mathfrak{s u}(2) \rightarrow \mathfrak{g}$. Every $\rho$ leads to an interesting theory, but to get the Jones polynomial and Khovanov homology, we take $\rho$ to be a principal embedding in the sense of Kostant. (For $G=S U(2)$, this simply means that $\rho$ is the identity map $\mathfrak{s u}(2) \rightarrow \mathfrak{g}$. For $G=S U(N)$, it means that the $N$-dimensional representation of $\mathfrak{g}$ is irreducible with respect to $\rho(\mathfrak{s u}(2))$.)

The solution I have just described is what I call the Nahm pole solution, since the relevant singularity was introduced long ago by Nahm in his work on magnetic monopoles [23]. That was in the context of "Nahm's equation," which is an ordinary differential equation for three $\mathfrak{g}$-valued functions $\phi_{1}, \phi_{2}, \phi_{3}$ of a real variable $y$ :

$$
\frac{\mathrm{d} \phi_{1}}{\mathrm{~d} y}+\left[\phi_{2}, \phi_{3}\right]=0, \text { and cyclic permutations. }
$$

The KW equations reduce to Nahm's equation if we drop the dependence on $\vec{x}$ and set $A=\phi_{y}=0$. On $\mathbb{R}^{3} \times \mathbb{R}_{+}$, the Nahm pole boundary condition just says that a solution is supposed to be asymptotic to the Nahm pole solution for $y \rightarrow 0$.

To state the Nahm pole boundary condition on $M_{4}=W_{3} \times \mathbb{R}_{+}$, for a more general 3-manifold $W_{3}$, one needs to specify some terms of $\mathcal{O}(1)$ in the solution (for $y \rightarrow 0$ ) as well as the singular terms of order $1 / y$. For $G=S U(2)$, one takes the $G$-bundle $E$ on which we are solving the equations to be, when restricted to $W_{3} \times\{y=0\}$, the frame bundle of $W_{3}$, so that ad $(E)=T W_{3}$. Then one takes $A$, restricted to the boundary, to be the Levi-Civita connection on $T W_{3}$. With this choice of $E$, the formula

$$
\phi=\sum_{i=1}^{3} \frac{\mathfrak{t}_{i} \cdot \mathrm{d} x_{i}}{y}
$$

makes sense (one can think of the numerator $\sum_{i} \mathfrak{t}_{i} \cdot \mathrm{d} x_{i}$ as stating the identification $\operatorname{ad}(E) \cong T W_{3}$ ). One can show that this choice of $(A, \phi)$ obeys the Nahm pole boundary condition up to an error 
of $\mathcal{O}(y)$, and the Nahm pole boundary condition simply says that the solution should agree with what I have described up to $\mathcal{O}(y)$. (One can generalize this to the case that the metric of $M_{4}$ is not a product near the boundary.)

Showing that the Nahm pole boundary condition is elliptic is mostly an exercise in "uniformly degenerate elliptic operators," but one needs to know some specific facts about the KW equations. The main thing that one needs to know is that if $\mathcal{L}$ is the linearization of the KW equations on a half-space $\mathbb{R}_{+}^{4}$ around the Nahm pole solution, then $\mathcal{L}$ as an operator between appropriate Hilbert spaces of functions on $\mathbb{R}_{+}^{4}$ has no kernel or cokernel. Actually one can show in an elementary way that $\mathcal{L}^{\dagger}=-N \mathcal{L} N^{-1}$ with an explicit matrix $N$, so it suffices to show that there is no kernel.

Much the same argument that proves this actually proves the following statement: The only solution of the KW equations on $\mathbb{R}_{+}^{4}$, approaching the Nahm pole solution for $y \rightarrow 0$ and also for $\sqrt{\vec{x}^{2}+y^{2}} \rightarrow \infty$, is the Nahm pole solution, and moreover this solution is "transverse" (ln expanding around it, the operator $\mathcal{L}$ has zero kernel and cokernel). In terms of Khovanov homology, this means that the Khovanov homology of the empty link is of rank 1.

Before trying to prove these vanishing results, I will explain a simpler vanishing result for the KW equations on a four-manifold $M=M_{4}$ without boundary. This will help us know what to aim for. ${ }^{3}$

The KW equations actually have many different useful Weitzenbock formulas. I will first state some formulas that are useful if we are on a manifold without boundary. Let $\mathcal{V}=F-\phi \wedge \phi-\star \mathrm{d}_{A} \phi$, $\mathcal{W}=\mathrm{d}_{A} \star \phi$, so the KW equations are $\mathcal{V}=\mathcal{W}=0$. Clearly then the KW equations are equivalent to the vanishing of

$$
I=-\int_{M} \operatorname{Tr}(\mathcal{V} \wedge \star \mathcal{V}+\mathcal{W} \wedge \star \mathcal{W})
$$

A short calculation gives

$$
I=-\int_{M} \mathrm{~d}^{4} x \sqrt{g} \operatorname{Tr}\left(\frac{1}{2} F_{i j} F^{i j}+D_{i} \phi_{j} D^{i} \phi^{j}+R_{i j} \phi^{i} \phi^{j}+\frac{1}{2}\left[\phi_{i}, \phi_{j}\right]\left[\phi^{i}, \phi^{j}\right]\right)
$$

with $R_{i j}$ the Ricci tensor. If $R_{i j}$ is non-negative, then this is a sum of non-negative terms. The condition $I=0$ forces all these terms to vanish and leads to only a rather trivial class of solutions.

But it is possible to say something useful even if $R_{i j}$ is not non-negative, because it is possible to find a family of Weitzenbock formulas. Define the selfdual and anti-selfdual two-forms $\mathcal{V}^{+}(t)=$ $\left(F-\phi \wedge \phi+t \mathrm{~d}_{A} \phi\right)^{+}, \mathcal{V}^{-}(t)=\left(F-\phi \wedge \phi-t^{-1} \mathrm{~d}_{A} \phi\right)^{-}$. The equations $\mathcal{V}^{+}(t)=\mathcal{V}^{-}(t)=\mathcal{W}=0$ are a 1-parameter family of elliptic equations, parametrized by $t \in \mathbb{R P}^{1}$. One finds that

$$
\begin{gathered}
-\int_{M} \mathrm{~d}^{4} x \sqrt{g} \operatorname{Tr}\left(\frac{t^{-1}}{t+t^{-1}} \mathcal{V}_{i j}^{+}(t) \mathcal{V}^{+i j}(t)+\frac{t}{t+t^{-1}} \mathcal{V}_{i j}^{-}(t) \mathcal{V}^{-i j}(t)+\mathcal{W}^{2}\right) \\
=I+\frac{t-t^{-1}}{4\left(t+t^{-1}\right)} \int_{M} \mathrm{~d}^{4} x \epsilon^{i j k l} \operatorname{Tr} F_{i j} F_{k l} .
\end{gathered}
$$

\footnotetext{
${ }^{3}$ The vanishing result without boundary was obtained in [15] and the case with a boundary was analyzed in [21].
} 
In other words, the same quantity $I$ can be written as a sum of squares in many different ways, modulo the topological invariant

$$
J(t)=\frac{t-t^{-1}}{4\left(t+t^{-1}\right)} \cdot 32 \pi^{2} P, \quad P=\frac{1}{32 \pi^{2}} \int_{M} \mathrm{~d}^{4} x \epsilon^{i j k l} \operatorname{Tr} F_{i j} F_{k l} .
$$

Now we can deduce the following: (1) The KW equations cannot have any solutions for $t \neq 0, \infty$ except with $P=0$ (if $P \neq 0$ for some solution, then by looking at the Weitzenbock formula at some value of $t^{\prime}$ with $J\left(t^{\prime}\right)<J(t)$, we reach a contradiction). And (2): If the KW equations are obeyed at one value of $t$ other than $0, \infty$, then they are obeyed at all $t$. This is an immediate consequence of the Weitzenbock formula, once we know that $P=0$. The equations then reduce to $\mathcal{F}=0$, where $\mathcal{F}=\mathrm{d} \mathcal{A}+\mathcal{A} \wedge \mathcal{A}$, with $\mathcal{A}$ the complex connection $\mathcal{A}=A+i \phi$, along with $\mathrm{d}_{A} \star \phi=0$. According to a theorem of Corlette [24], the solutions correspond to homomorphisms $\pi_{1}(M) \rightarrow G_{\mathbb{C}}$ that are in a certain sense semi-stable.

The moral of the story is that the KW equations participate in many different Weitzenbock formulas, not just one, and it is important to know all of them. However, none of the formulas that I have written down so far are useful for understanding the Nahm pole boundary condition. The reason is that if $\partial M \neq \varnothing$, then the preceding formulas (whose derivation involves integration by parts) will have boundary contributions if we are on a manifold with boundary, and those boundary contributions are divergent in the case of a solution with Nahm pole boundary behavior. This is inevitable because the expression that I called $I$ in writing the Weitzenbock formula is divergent in the case of a solution with Nahm pole behavior. A formula like

$$
-\int \operatorname{Tr}(\mathcal{V} \wedge \star \mathcal{V}+\mathcal{W} \wedge \star \mathcal{W})=I+\text { boundary correction }
$$

must have a boundary correction $-\infty$ in the case of a Nahm pole, since the left hand side is 0 and $I=+\infty$. A Weitzenbock formula with such divergent terms is not likely to be useful.

To get around this, the best we could hope for would be a Weitenbock formula on $\mathbb{R}_{+}^{4}$ in which $I$ is replaced by a sum of squares of quantities whose vanishing characterizes the Nahm pole solution $A=0, \phi=\overrightarrow{\mathfrak{t}} \cdot \mathrm{d} \vec{x} / y$. The quantities that vanish ${ }^{4}$ in the Nahm pole solution are the curvature $F$, covariant derivatives and commutators that involve $\phi_{y}$, namely $D_{i} \phi_{y}$ and $\left[\phi_{i}, \phi_{y}\right]$, covariant derivatives of $\phi_{b}$ along $\mathbb{R}^{3}$ such as $D_{a} \phi_{b}$, and finally $W_{a}=D_{y} \phi_{a}+\frac{1}{2} \varepsilon_{a b c}\left[\phi_{b}, \phi_{c}\right]$. (Nahm's equation is $W_{a}=0$.) What we need is true. Define the following sum of squares of the objects whose vanishing characterizes the Nahm pole solution:

$$
\begin{aligned}
I^{\prime}=-\int_{\mathbb{R}^{3} \times \mathbb{R}_{+}} \mathrm{d}^{4} x \operatorname{Tr}\left(\frac{1}{2} \sum_{i, j} F_{i j}^{2}\right. & +\sum_{a, b}\left(D_{a} \phi_{b}\right)^{2}+\sum_{i}\left(D_{i} \phi_{y}\right)^{2} \\
& \left.+\sum_{a}\left[\phi_{y}, \phi_{a}\right]^{2}+\sum_{a} W_{a}^{2}\right) .
\end{aligned}
$$

\footnotetext{
${ }^{4}$ In the following, indices $i, j$ take four values corresponding to $x_{1}, x_{2}, x_{3}, y$, but indices $a, b$ take only three values corresponding to $x_{1}, x_{2}, x_{3}$. Also, $\varepsilon_{a b c}$ is the Levi-Civita antisymmetric tensor.
} 
Then there is an identity along the lines that we need:

$$
-\int_{M} \operatorname{Tr}\left(\mathcal{V}^{2}+\mathcal{W}^{2}\right)=I^{\prime}+\Delta
$$

where $\Delta$ is a certain boundary term

$$
\Delta=-\int_{\partial \mathbb{R}_{+}^{4}} \operatorname{Tr}(\phi \wedge F+\ldots) .
$$

(I have omitted some further terms in $\Delta$.) $\Delta$ is the sum of a contribution at the boundary $y=0$ and on a large hemisphere $\sqrt{\vec{x}^{2}+y^{2}}>>1$.

Now to get a vanishing theorem that will say that the global Nahm pole solution is the only solution on $\mathbb{R}_{+}^{4}$ that obeys Nahm pole boundary conditions, we need to do the following. We have to prove that if $A, \phi$ approach the Nahm pole solution for $y \rightarrow 0$ and for $\sqrt{\vec{x}^{2}+y^{2}} \rightarrow \infty$, then they approach it fast enough so that $\Delta=0$. Once this is established, the Weitzenbock formula will say that a KW solution that satisfies the boundary condition must have $I^{\prime}=0$. But $I^{\prime}$ was constructed so that it vanishes for and only for the Nahm pole solution.

To find the expected behavior of a solution for $y \rightarrow 0$ is a matter of looking at an ODE in which one ignores the $\vec{x}$ dependence, since that is nonsingular. In effect, then, we just have to look at the eigenvalues of the linearization of Nahm's equation (or more exactly a doubled version of Nahm's equation with $A$ as well as $\phi$ ). Half of the linearized eigenvalues are negative and half are positive. The Nahm pole boundary condition amounts to setting to 0 the coefficients of perturbations with negative eigenvalues, and allowing the positive ones. The positive eigenvalues are large enough to ensure that $\Delta=0$ when the Nahm pole boundary condition is obeyed.

This shows that there is no contribution to $\Delta$ from the boundary at $y=0$. To show that there is no contribution to $\Delta$ at $\sqrt{\vec{x}^{2}+y^{2}} \rightarrow \infty$, one needs to look at the eigenvalues of the "angular" part of the operator $\mathcal{L}$, which is an operator on a hemisphere $S_{+}^{3}$ with Nahm pole boundary conditions along the boundary. Those eigenvalues determine how fast a solution will vanish at infinity, assuming that it does vanish at infinity. Again the spectrum is such that there is no contribution to $\Delta$.

This leads to the nonlinear vanishing theorem - the global Nahm pole solution is the only solution on the half-space that satisfies the boundary conditions. Much the same argument proves a linearization of the same statement: the operator $\mathcal{L}$ obtained by linearizing around the Nahm pole solution has trivial kernel (and hence also trivial cokernel, since $\mathcal{L}$ is conjugate to $-\mathcal{L}^{\dagger}$ ).

Together with the machinery of uniformly degenerate elliptic operators, this leads to the ellipticity of the Nahm pole boundary condition in the absence of knots. But what are we supposed to say in the presence of knots? As already noted, the knot will be in the boundary (fig. 10). To incorporate a knot in the boundary, we introduce a refinement of the Nahm pole boundary condition, such that $(A, \phi)$ obeys the Nahm pole boundary condition at a generic boundary point away from a knot, but has some more subtle behavior near the knot.

To describe what this more subtle behavior should be, we consider the case that the knot is 


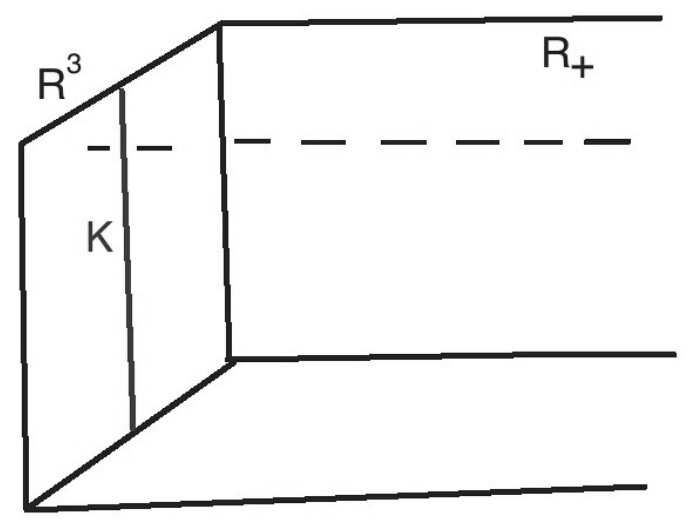

Figure 11: A model situation in which a knot is represented by a straight line $K=\mathbb{R}$ in the boundary of a half-space $\mathbb{R}_{+}^{4}$.

locally a straight line $\mathbb{R} \subset \mathbb{R}^{3}$, so we work on $\mathbb{R}_{+}^{4}$ with a knot that lives on a straight line $K$ in the boundary (fig. 11).

The idea is going to be to find a singular model solution in the presence of the knot. This solution will coincide with the Nahm pole solution near a boundary point away from $K$, but it will look different near a point of $K$. The model solution will depend on the choice of an irreducible representation $R^{\vee}$ of the dual group $G^{\vee}$. Then a boundary condition is defined by saying that one only allows solutions of the KW equations that look like the model solution near a knot.

For this to make sense, the model solution must look the same near any point of $K$, so we assume that the model solution is invariant under translations along $K$. So we reduce to equations on $\mathbb{R}^{2} \times \mathbb{R}_{+}$with the knot now represented by a point $p \in \mathbb{R}^{2}$ (fig. 12).

Once we reduce to 3 dimensions (and assume vanishing of $A_{1}$ and $\phi_{y}$ in a way that can be motivated by the Weitzenbock formula) the $\mathrm{KW}$ equations become tractable. Pick coordinates so that $x_{1}$ runs along the knot $K ; x_{2}, x_{3}$ parametrize the normal plane to $K$ in the boundary; and $y$ measures the distance from the boundary. Define the three operators

$$
\begin{aligned}
& \mathcal{D}_{1}=D_{2}+i D_{3}=\frac{\partial}{\partial x_{2}}+i \frac{\partial}{\partial x_{3}}+\left[A_{2}+i A_{3}, \cdot\right] \\
& \mathcal{D}_{2}=D_{y}-i\left[\phi_{1}, \cdot\right]=\frac{\partial}{\partial y}+\left[A_{y}-i \phi_{1}, \cdot\right] \\
& \mathcal{D}_{3}=\left[\phi_{2}-i \phi_{3}, \cdot\right],
\end{aligned}
$$

and also the "moment map"

$$
\mu=F_{23}-\left[\phi_{2}, \phi_{3}\right]-D_{y} \phi_{1} .
$$




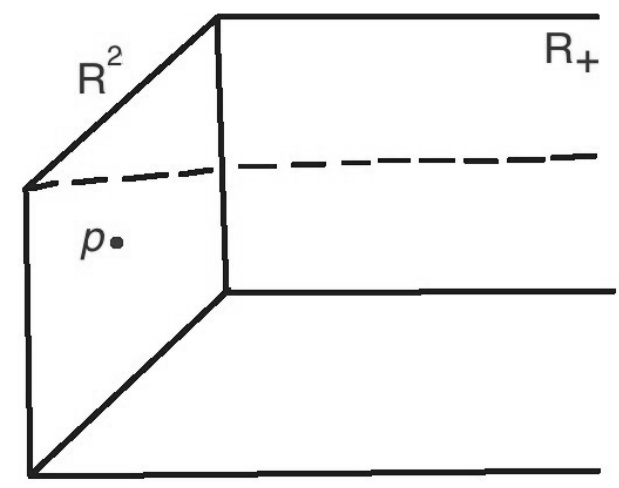

Figure 12: Assuming translation invariance along the $K$ direction in fig. 11, we reduce to $\mathbb{R}_{+}^{3}=\mathbb{R}^{2} \times \mathbb{R}_{+}$, with the knot now represented by a point $p$ in the boundary.

The KW equations in this situation become

$$
0=\left[\mathcal{D}_{i}, \mathcal{D}_{j}\right], \quad i, j=1, \ldots, 3
$$

along with a "moment map" condition

$$
\mu=0 .
$$

These equations were introduced in [15] and were called the extended Bogomolny equations. They are a sort of hybrid of three much-studied equations in the mathematics of gauge theory. If we drop $\mathcal{D}_{1}$ (by assuming that the fields are independent of $x_{2}$ and $x_{3}$ and that $A_{2}=A_{3}=0$ ), we get Nahm's equation; if we drop $\mathcal{D}_{2}$ (by assuming that the fields are independent of $y$ and that $A_{y}=\phi_{1}=0$ ) we get Hitchin's equation; and if we drop $\mathcal{D}_{3}$ (by setting $\phi_{2}=\phi_{3}=0$ ), we get the Bogomolny equations.

The full system of equations is tractable for the same reason each of those three specializations is. There are two key facts: (a) the equations $\left[\mathcal{D}_{i}, \mathcal{D}_{j}\right]=0$ are invariant under $G_{\mathbb{C}}$-valued gauge transformations $\left(G_{\mathbb{C}}\right.$ is the complexification of $\left.G\right)$; (b) the combination of setting $\mu=0$ and dividing by $G$-valued gauge transformations is equivalent to forgetting the condition $\mu=0$ and dividing by

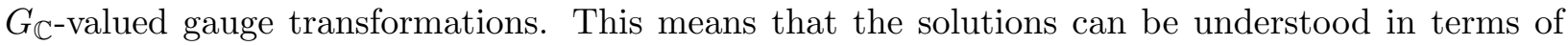
complex geometry.

It is reasonable to expect that the model solution possesses the symmetries of the knot. So we assume that the model solution is invariant under a rotation of the boundary $\mathbb{R}^{2}$ around the point $p \in \mathbb{R}^{2}$ at which the knot lives, and also invariant under a scaling of $\mathbb{R}^{2} \times \mathbb{R}_{+}$keeping $p$ fixed. With these assumptions, the equations $\left[\mathcal{D}_{i}, \mathcal{D}_{j}\right]=\mu=0$ reduce to affine Toda equations which are integrable. One can find all the solutions in closed form, and the solutions that satisfy the Nahm pole boundary condition away from the knot are classified by an irreducible representation $R^{\vee}$ of the dual group $G^{\vee}$. These solutions were found for $G=S O(3), G^{\vee}=S U(2)$ in [3] and more generally in [25]. 
How would one go about proving that the KW equations with a boundary condition defined by one of these model equations is a well-posed (elliptic) problem? Basically, modulo generalities about uniformly degenerate elliptic operators, we need to show that the operator $\mathcal{L}$ obtained by linearizing around one of these solutions has no kernel or cokernel. It is again sufficient to show that the kernel vanishes, since $\mathcal{L}^{\dagger}$ is conjugate to $-\mathcal{L}$.

Just as in the absence of a knot, we will actually find a nonlinear analog of the vanishing of the kernel of $\mathcal{L}$ : any solution of the KW equations on $\mathbb{R}^{3} \times \mathbb{R}_{+}$(with the knot as an infinite straight line in the boundary, as before) that is asymptotic to the model solution both along the boundary and at infinity actually coincides with it.

The vanishing results we want are the sort that often follow from a Weitzenbock formula. But none of the Weitzenbock formulas that we considered before are well-adapted to the presence of a knot. Even the more subtle Weitzenbock formula that includes the Nahm pole singularity away from a knot

$$
-\int_{M} \operatorname{Tr}\left(\mathcal{V}^{2}+\mathcal{W}^{2}\right)=I^{\prime}+\Delta
$$

does not give any useful information, because $I^{\prime}$ (which is the sum of squares of quantities that vanish in the Nahm pole solution without a knot) is divergent in the presence of a knot so $I^{\prime}$ will be $+\infty$ and hence $\Delta$ will be $-\infty$ with a knot present.

So we need a new Weitzenbock formula. We imitate what we did before. We find a collection of quantities $X_{i}$ whose vanishing characterizes the model solution. (Some obvious $X_{i}$ are real and imaginary parts of $\left[\mathcal{D}_{i}, \mathcal{D}_{j}\right]$, and also $\mu$; the others are quantities like $\left[\phi_{i}, \phi_{y}\right]$ that vanish because the model solution has $A_{1}=\phi_{y}=0$.) Then if

$$
I^{\prime \prime}=-\sum_{i} \int_{\mathbb{R}^{3} \times \mathbb{R}_{+}} \operatorname{Tr} X_{i}^{2}
$$

we have to hope that there is an identity

$$
-\int_{\mathbb{R}^{3} \times \mathbb{R}_{+}} \operatorname{Tr}(\mathcal{V} \wedge \star \mathcal{V}+\mathcal{W} \wedge \star \mathcal{W})=I^{\prime \prime}+\widehat{\Delta}
$$

where $\widehat{\Delta}$ is a new boundary term. It turns out that there is indeed an identity like this.

We still need to show that $\widehat{\Delta}=0$ in the case of a solution that obeys the KW equations and is asymptotic near the knot to the model solution. For this, one needs to know what is the asymptotic behavior near the boundary and at infinity of a solution of the KW equations. We already know the behavior at a generic boundary point, which was used in our proof of the well-posedness of the Nahm pole boundary condition without a knot. To find the behavior near the knot and also at infinity, we now need to solve the angular part of the equation on a $2 \mathrm{~d}$ hemisphere $S_{+}^{2}$ (fig. 13).

Again it turns out that the eigenvalues of the angular operator are favorable, so there is no contribution to $\widehat{\Delta}$ either near $p$ or at infinity. This fact together with the relevant Weitzenbock formula imply that a solution of the $\mathrm{KW}$ equations on $\mathbb{R}^{3} \times \mathbb{R}_{+}$that is asymptotic on the boundary 


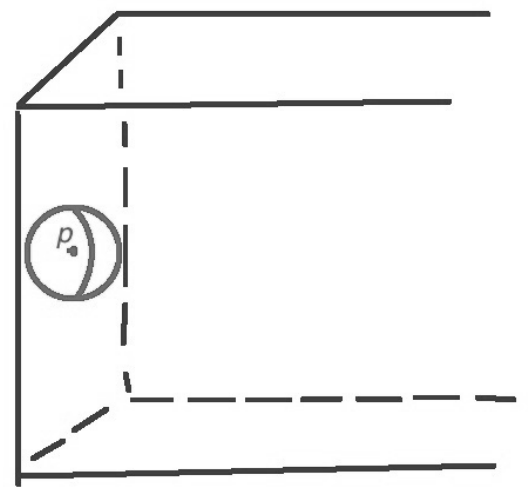

Figure 13: To prove the vanishing theorem in the presence of a knot, one has to study the angular operator near a singular point on the boundary. The angular operator is defined on the indicated hemisphere.

and at infinity to the model solution with the knot actually coincides with that model solution. A linearized version of the same argument shows that the kernel of $\mathcal{L}$ vanishes, which is what we actually needed to know for ellipticity.

This is the main step in showing that $\mathcal{L}$ is a Fredholm operator in the presence of an arbitrary knot $K$ embedded in any three-manifold $W$. Some details are still needed to show that this gives an elliptic boundary condition for the nonlinear $\mathrm{KW}$ equations in the general case of a curved knot [22].

\section{A Some Physics Background}

In this appendix, I will briefly describe some physics background to the treatment of singly-graded Khovanov homology in Lecture One. Only a bare outline of the string/M-theory context and the framework of [3] for doubly-graded Khovanov homology will be given. I aim primarily to explain what is different for the singly-graded theory.

The starting point is the existence of a six-dimensional superconformal field theory with $(0,2)$ supersymmetry, associated to any simply-laced Lie group $G$, or more precisely to its Dynkin diagram. This theory has a $\operatorname{Spin}(5)$ group of R-symmetries. Making use of a subgroup $F=\operatorname{Spin}(2) \subset \operatorname{Spin}(5)$, the theory can be topologically twisted in such a way that it can be compactified on a Riemann surface $C$ to give a four-dimensional theory with $\mathcal{N}=2$ supersymmetry. These are the theories of class $\mathrm{S}$, as studied in [26]. They have an R-symmetry group $\widehat{F}=(\operatorname{Spin}(3) \times \operatorname{Spin}(2)) / \mathbb{Z}_{2} \cong \mathrm{U}(2)$ (the subgroup of $\operatorname{Spin}(5)$ that commutes with $F$ ). Using the Spin(3) factor, a theory of class $\mathrm{S}$ can be topologically twisted and compactified on a four-manifold $M$ in a way that preserves one supercharge $Q$. This gives a theory on $M \times C$ that is topological along $M$ and holomorphic along $C$. (The holomorphy along $C$ means, for example, that the cohomology of $Q$ acting on local operators 
on $M \times C$ varies homomorphically on $C$.) The topological-holomorphic theory on $M \times C$ still has an $F=\operatorname{Spin}(2)$ symmetry, because $F$ commutes with the group $\widehat{F}$ that was used in the twisting.

The underlying six-dimensional $(0,2)$ model admits half-BPS surface operators that can be supported on any two-manifold $U$ in six dimensions. However, when the theory is formulated on $M \times C$ as summarized in the last paragraph, if we wish to preserve the supercharge $Q$ of the topological-holomorphic theory, the possible choices of $U$ are quite limited. $U$ must be of the form $p \times C$, where $p$ is a point in $M$, or $\Sigma \times q$, where $\Sigma \subset M$ is a two-manifold and $q$ is a point in $C$. The reason for this is essentially that any (complete, connected) complex submanifold of $C$ is $C$ itself or a point $q \in C$. For constructing Khovanov homology, we take $U=\Sigma \times q$.

To get singly-graded Khovanov homology, we take $M$ to be simply $\mathbb{R}^{4}$, and $C$ to be a cylinder $\mathbb{R} \times S^{1}$. The six-manifold $M \times C$ is then simply $\mathbb{R}^{4} \times \mathbb{R} \times S^{1}$. The supercharge $Q$ is invariant under rotations of $\mathbb{R}^{4}$ (combined with a suitable element of $\operatorname{Spin}(3) \subset \widehat{F}$ ) but not under more general rotations of $\mathbb{R}^{4} \times \mathbb{R}$. Now we use the fact that the $(0,2)$ model, when formulated on $M^{\prime} \times S^{1}$ for any five-manifold $M^{\prime}$, with the radius of $M^{\prime}$ being much greater than that of $S^{1}$, reduces at long distances on $M^{\prime}$ to maximally supersymmetric gauge theory, with gauge group $G$. In the context of the topological-holomorphic theory described above, this reduction is valid without taking any large distance limit. The resulting supersymmetric gauge theory on $M^{\prime}=\mathbb{R}^{4} \times \mathbb{R}$ is infrared-free (in sharp contrast to the underlying $(0,2)$ model in six dimensions) and can be analyzed by classical methods. In particular, the condition for $Q$-invariance becomes the HW equations, which were mentioned in Lecture One. These are equations for a pair consisting of a gauge field $A$, and a field $B$ on $\mathbb{R}^{4} \times \mathbb{R}$ that is an adjoint-valued section of the pullback to $\mathbb{R}^{4} \times \mathbb{R}$ of the bundle of selfdual two-forms on $\mathbb{R}^{4}$.

A surface operator in six dimensions supported on $\Sigma \times q$ reduces in the gauge theory description to an 't Hooft-like surface operator supported on $\Sigma \times q^{\prime}$, where $q \in \mathbb{R} \times S^{1}$ projects to $q^{\prime} \in \mathbb{R}$. A solution of the HW equations in the presence of this surface operator is supposed to have a singularity along $\Sigma \times q^{\prime}$. This codimension three singularity should be modeled on the standard codimension three singularity of the Bogomolny equations, suitably embedded in the HW equations with gauge group $G$.

To categorify the quantum knot invariants associated to a representation $R^{\vee}$ of a simply-laced ${ }^{5}$ compact Lie group $G^{\vee}$, one studies the HW equations with gauge group $G$ (the Langlands-GNO dual of $G^{\vee}$, which in particular has the same Lie algebra as $G^{\vee}$ if $G$ is simply-laced), and with the appropriate singularity along $\Sigma \times q^{\prime}$. The appropriate singularity is obtained, as discussed in Lecture One, by embedding a singular $U(1)$ solution in $G$ using the homomorphism $\rho: \mathfrak{u}(1) \rightarrow \mathfrak{t} \subset \mathfrak{g}$ that is dual to $R^{\vee}$.

The HW equations on $\mathbb{R}^{4} \times \mathbb{R}$ are compatible with the familiar codimension three singularity on a two-manifold $V \subset \mathbb{R}^{4} \times \mathbb{R}$ if and only if $V$ is of the form $\Sigma \times q^{\prime}$ with $\Sigma \subset \mathbb{R}^{4}, q^{\prime} \in \mathbb{R}$. This statement is easily verified by inspection of the HW equations. The explanation that we have given here starting with the $(0,2)$ model serves to explain "why" it is true. The restriction to

\footnotetext{
${ }^{5}$ If $G^{\vee}$ is not simply-laced, one requires a refinement described in section 5.5 of [3].
} 
$V \subset \mathbb{R}^{4} \times q^{\prime} \subset \mathbb{R}^{4} \times \mathbb{R}$ is completely essential for getting knot theory out of this construction, since the relevant topology would disappear if $V$ were free to move in five dimensions. For example, Khovanov homology arises in the "time"-independent case $\Sigma=\mathbb{R}_{t} \times K \subset \mathbb{R}_{t} \times \mathbb{R}^{3}=\mathbb{R}^{4}$, where $K$ is a knot in $\mathbb{R}^{3}$ and the first factor in $\mathbb{R}^{4}=\mathbb{R}_{t} \times \mathbb{R}^{3}$ is parametrized by the "time." If $V$ were free to vary in $\mathbb{R}^{4} \times \mathbb{R}$, then $K$ would be free to vary in $\mathbb{R}^{3} \times \mathbb{R}$ (the product of the last two factors in $\mathbb{R}^{4} \times \mathbb{R}=\mathbb{R}_{t} \times \mathbb{R}^{3} \times \mathbb{R}$ ), and could be trivially unknotted. Much the same point was made in Lecture One. A more general $V$ (not of the time-independent form $\mathbb{R}_{t} \times K$ ) is used to define the "morphisms" of Khovanov homology.

What we have described corresponds to the singly-graded version of Khovanov homology (with only the cohomological grading and no " $q$ "-grading). The single grading comes from the symmetry group $F \cong \operatorname{Spin}(2)$ that was maintained throughout the construction. To get doubly-graded Khovanov homology, one takes $C$ to be not $\mathbb{C}^{*}=\mathbb{R} \times S^{1}$ but $\mathbb{C}$. This is done by adding to $\mathbb{C}^{*}$ a point $q_{0}$ "at infinity." $\mathbb{C}$ admits an $S^{1}$ action, leaving fixed the point $q_{0}$. In the underlying $(0,2)$ model, one considers a surface operator supported on $U=\Sigma \times q_{0}$. Reduction of $M \times C$ on the orbits of $S^{1}$ leads now to a description in terms of gauge theory on $M \times \mathbb{R}_{+}$(where $\mathbb{R}_{+}$, a half-line, is the quotient $C / S^{1}$ ). The $S^{1}$ action leads to the desired second grading. This doubly-graded version of the construction was the main subject of [3], and the details will not be repeated here.

Research supported in part by NSF Grant PHY-1314311. I thank M. Abouzaid, C. Manolescu, and R. Mazzeo for discussions.

\section{References}

[1] S. Gukov, A. S. Schwarz, and C. Vafa, "Khovanov-Rozansky Homology And Topological Strings," Lett. Math. Phys. 74 (2005) 53-74, hep-th/0412243.

[2] H. Ooguri and C. Vafa, "Knot Invariants And Topological Strings," Nucl. Phys. B577 (2000) 419, hep-th/9912123.

[3] E. Witten, "Fivebranes And Knots," Quantum Topology 3 (2012) 1-137, arXiv:1101.3216.

[4] E. Witten, "Khovanov Homology and Gauge Theory," in R. Kirby, V. Krushkal, and Z. Wang, eds., Proceedings Of The FreedmanFest (Mathematical Sciences Publishers, 2012) 291-308, arXiv:1108.3103.

[5] E. Witten, "Two Lectures On The Jones Polynomial And Khovanov Homology," arXiv:1401.6996.

[6] P. Seidel and I. Smith, "A Link Invariant From The Symplectic Geometry of Nilpotent Slices," Duke Math. J. 134 (2006) 453-514.

[7] C Manolescu, "Nilpotent Slices, Hilbert Schemes, and the Jones Polynomial," Duke Math. J. 132 (2006) 311-369.

[8] M. Abouzaid and I. Smith, "Khovanov Homology From Floer Cohomology," arXiv:1504.01230. 
[9] V. F. R. Jones, "A Polynomial Invariant For Knots Via Von Neumann Algebra," Bull. Am. Math. Soc. 12 (1985) 103-11.

[10] M. F. Atiyah, "Floer Homology," Progr. Math. 133 (1995) 105.

[11] D. Gaiotto and E. Witten, "Knot Invariants From Four-Dimensional Gauge Theory," Adv. Theor. Math. Phys. 16 (2012) 935, arXiv:1106.4789.

[12] J. Kamnitzer, "The Beilinson-Drinfeld Grassmannian and Symplectic Knot Homology," in D. A. Ellwood (ed.), Grassmannians, Moduli Spaces, and Vector Bundles (Clay Math Institute, Cambridge MA and American Mathematical Society, Providence RI, 2011) 81-94.

[13] S. Cautis and J. Kamnitzer, "Knot Homology Via Derived Categories Of Coherent Sheaves, I. The $\mathfrak{s l}(2)$ Case," Duke Math. J. 142 (2008) 511-588.

[14] G. 't Hooft, "On The Phase Transition Towards Permanent Quark Confinement," Nucl. Phys. B138 (1978) 1-25.

[15] A. Kapustin and E. Witten, "Electric-Magnetic Duality And The Geometric Langlands Program," Commun. Numb. Th. Physics 1 (2007) 1-236.

[16] S. Bigelow, "A Homological Definition Of The Jones Polynomial," Geometry and Topology Monographs, Volume 4: Invariants of Knots and 3-Manifolds (Kyoto, 2001), pp. 29-41.

[17] R. Lawrence, "A Functorial Approach to the One-Variable Jones Polynomial," J. Diff. Geom. 37 (1993) 689-710.

[18] D. Galakhov and G. W. Moore, "Comments On The Two-Dimensional Landau-Ginzburg Approach," arXiv:1607.04222.

[19] A. Haydys, "Fukaya-Seidel Category And Gauge Theory," J. Symp. Geom. 13 (2015) 151-207, arXiv:1010.2353.

[20] S. Gukov, P. Putrov, and C. Vafa, "Fivebranes and 3-manifold Homology," arXiv:1602.05302.

[21] R. Mazzeo and E. Witten, "The Nahm Pole Boundary Condition," in The Influence Of Solomon Lefschetz in Geometry and Topology, ed. L. Katzarkov et. al. (American Mathematical Society, 2014), arXiv:1311.3167.

[22] R. Mazzeo and E. Witten, "The Nahm Pole Boundary Condition With Knots," to appear.

[23] W. Nahm, "All Selfdual Multimonopoles for Arbitrary Gauge Groups" (NATO ASI, 1983).

[24] K. Corlette, "Flat G-Bundles With Canonical Metric," J. Diff. Geom. 28 (1988) 361-82.

[25] V. Mikhaylov, "On The Solutions Of Generalized Bogomolny Equations," JHEP 05 (2012) 112, arXiv:1202.4848.

[26] D. Gaiotto, “N = 2 Dualities," JHEP 1208 (2012) 034, arXiv:0904.2715. 